\title{
USING OF GSM LOCATOR IN SECURITY APPLICATIONS
}

\author{
Milan Adamek, Petr Neumann, Martin Pospisilik, Miroslav Matysek \\ Faculty of Applied Informatics, Tomas Bata University in Zlin, Nad Stranemi 4511, 76005 Zlin, Czech Republic
}

\begin{abstract}
This work deals with the construction of a locator to determine locations using GSM technology. The locator's design will be tailored especially for the mobile security of more expensive items. The theoretical part will discuss different ways of positioning - using GSM technology in detail. In the conclusion, the benefits of GSM localisation for security applications are evaluated and compared to satellite locations.
\end{abstract}

Keywords: GSM; mobile security; satellite
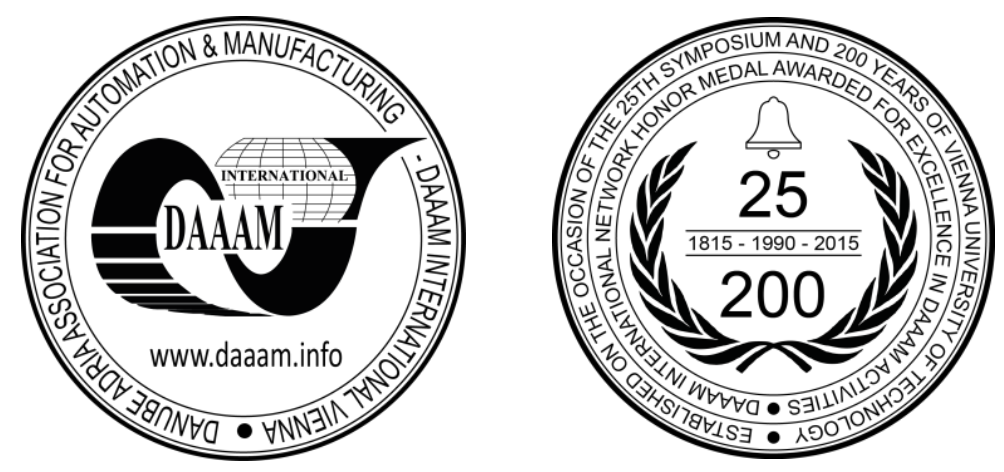

This Publication has to be referred as: Adamek, M[ilan]; Neumann, P[etr]; Pospisilik, M[artin] \& Matysek, M[iroslav] (2016). Using of GSM Locator in Security Applications, Proceedings of the 26th DAAAM International Symposium, pp.0075-0081, B. Katalinic (Ed.), Published by DAAAM International, ISBN 978-3-902734-07-5, ISSN 1726-9679, Vienna, Austria DOI: $10.2507 / 26$ th.daaam.proceedings.011 


\section{Introduction}

Mobile communications were used above all for maritime transport and had two basic differing features from the existing networks. The greatest drawback was the mutual incompatibility of individual networks; while the second distinct feature was (the use of) the analogue technologies that were used for the operation of these networks. GSM networks are commonly used for voice-calls, the transmission of messages in the form of SMS, and data transmissions. The aim of this paper is to exploit other GSM network services - and that is, for the determination of a position in the field. Unlike GPS technology, line-of-sight with satellites is unnecessary in order to determine location, thus GPS technology can also be used in places where satellites are not available to determine a required location. A proposed GPS locator is presented within the framework of this paper, and mention is made of inaccuracies in the course of determining positions.

\section{GSM localisation}

The main assumption of GSM localisation is the deployment of base stations across the whole structure of the GSM network. The area is divided into cells, whereby one cell is served by one BTS station or the BTS station is located at the interface and serves multiple cells - in such cases, we talk about sectors. The radius and the cell density are dependent on the estimated number of active mobile devices, or the population density in the given area. The number of BTS stations significantly affects the accuracy of the determination of a location [1].

Mobile stations communicate at any one instant with just one BTS station, but have information about other available BTS stations. BTS stations have a fixed location and are stationary, unlike mobile devices [6].

The following two identifiers important are for localisation:

- The cell ID is the unique identifier of the BTS base station.

- The Timing Advance (TA) is the parameter representing the signal propagation time between the mobile device and the network.

\subsection{Cell ID}

Each cell in a network has its own unique identifier. Its uniqueness is ensured within the framework of an operator in the given state. When logging into the network, the the mobile device can directly determine its location. Accuracy depends on the size of the area covered by the BTS station. Cell Sector is used for more accurate sectorisation - where the cell is divided into three sectors, which allows a two-thirds greater degree of precision [7].

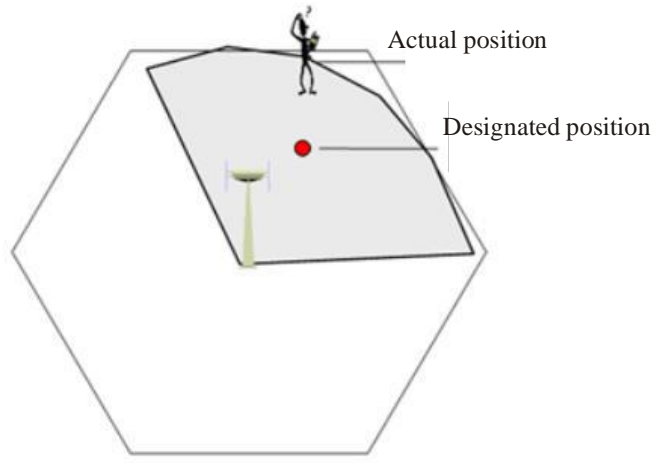

Fig. 1. The Cell ID [5]

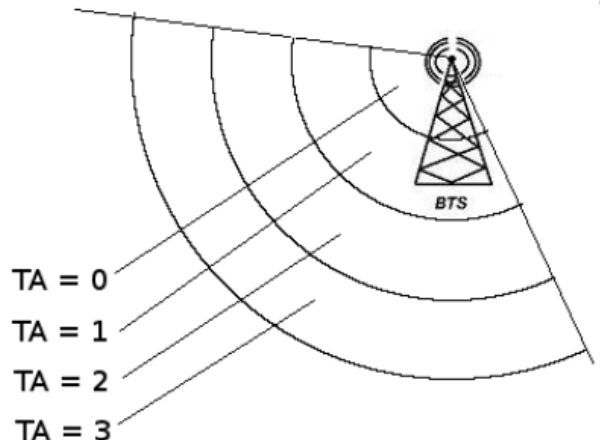

Fig. 2. TA identifier [5]

The estimated location of the mobile device is located in the centre of the field so created. TDMA specification requires that the chain manages to travel to the BTS station and back within half of the reserved $577 \mu$ s (microseconds). After deducting time caused by losses and signal quality, 233 microseconds remain. Within this time, the signal has to travel to the BTS station and back. If we multiply this radio signal speed data, we find that the signal may cover a track of up to 70,000 meters. This also sets the maximum radius of the BTS to $35 \mathrm{~km}$ [3].

\subsection{TA Timing advance}

The TA Identifier gives us the delay value between the mobile device and the BTS station. The value can be determined directly during a telephone call, after activation of the service menu, with values from 0 to 63 . The higher the value, the farther one is from the BTS station with which it is communicating. This parameter is stored in 4 bits, and if one multiplies this value by $547 \mathrm{~m}$, we can ascertain the distance from the BTS station [7]. 


\section{GSM localisation methods}

With this knowledge of the important parameters of the GSM network and an adequate list of the greatest possible number of BTS stations, we can perform localisation itself by exploiting the GSM network.

\subsection{Cell of origin}

Cell of Origin (COO) is a method based on the cellular structure of the GSM network. The top priority for this method is the Cell ID parameter, hence the accuracy of this method. In densely populated areas, the tolerance is about 200 meters; in less populated areas, the tolerance can theoretically reach up to 35 kilometers [6].

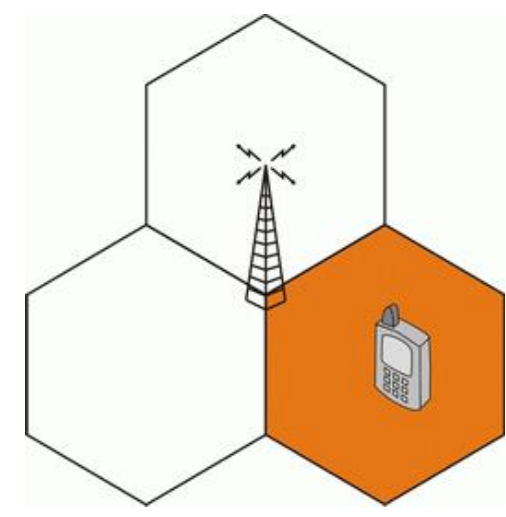

Fig. 3. The COO method [8]

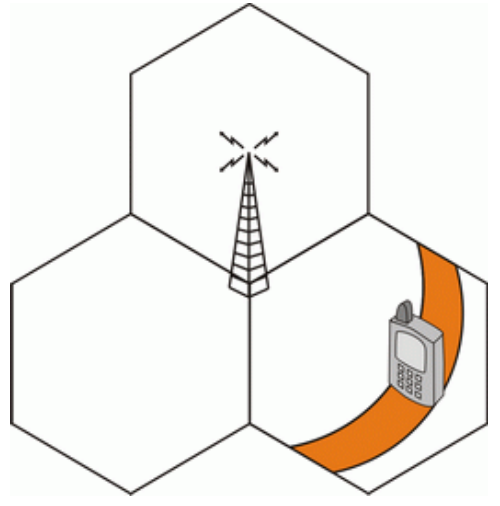

Fig. 4. TA method [3]

\subsection{The TA method}

TA is a method based on the knowledge of the distance of a mobile device from at least three BTS stations. For the method to work, it is necessary to adjust the mobile device such that it will know how to provide the requested information. Usually, it is sufficient to upload new firmware or use the SIM Toolkit application. When properly used, we can refine tolerance down to 125 meters [7].

\subsection{Time-of-arrival}

Time-Of-Arrival (TOA) is a method based on the TA method. Its basis is the knowledge of the distances from three BTS stations. This information however, is obtained from the BTS base stations. For this, we need mutual synchronisation - which does not normally occur. The method requires additional investments; while in urban areas above all, it does not produce better results than the COO method [6].

\subsection{Enhanced cell global identity}

Enhanced Cell Global Identity (E-CGI) is a method that complements and rounds out the Cell of Origin and Timing advance methods for measuring signal levels. The E-CGI method is used for calculating the distance of the mobile device from the BTS base-station. Areas with the most likely occurrence are identified according to the measured signal levels at the site/location of the mobile device and the knowledge of the transmission performance of the BTS base stations. The accuracy of this method is around 50-550 meters for densely populated areas, and $250 \mathrm{~m}$ to 8 $\mathrm{km}$ in rural areas with lower populations [9].

\subsection{Enhanced observed time difference}

The Enhanced Observed Time Difference (E-OTD) method is based on the installation of Location Measurement Unit (LMU) reference receivers within the context of the whole GSM network.

\section{GSM locator realisation}

In the GSM locator design process, priority was given to two basic requirements - namely, the requirement for small dimensions of the locator, and the requirement for long battery life. The Cell of Origin (COO) method was used for localisation by the locator. 


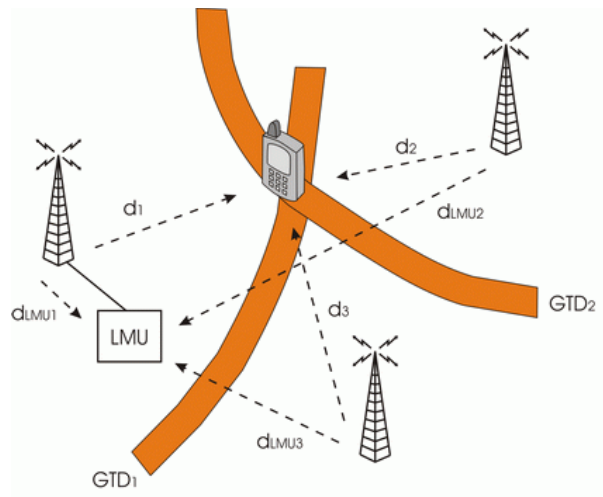

Fig. 5. The E-OTD method [4]

\subsection{The GSM module}

The basis of the whole system is the GSM module in which the SIM card is placed. The locator uses the smallest GSM module on the market from Enfora - the Enabler IIIG, type: GSM0308. The module is functional both in Europe and in the USA. It does not have a screen, microphone or keyboard - there is no need for these components [10]. The module can communicate across all GSM wave-bands, including GPRS data transmission. It includes a small antenna. Energy consumption, when demanded, is around $230 \mathrm{~mA}$ and only $10 \mathrm{~mA}$ in idle mode. These values are very low and contribute to extending the overall device battery life [11].

\subsection{The control module}

The PCB for controlling the circuit includes an interface for communicating with the quad-band GSM/GPRS module, microcontroller and microchip, as well as an on/off switch and an SOS button.

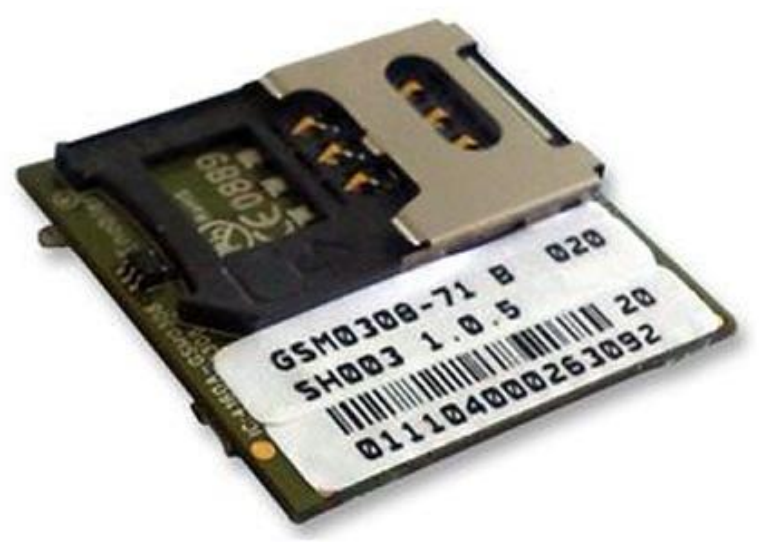

Fig. 6. The GSM Module [12]

\subsection{The operating principle of the GSM locator}

The control circuit is programmed in a loop that waits for a certain event. This event initialises the entire programme and it starts to search for the position. Such events may include the following:

- Incoming phone calls. In this case, the device hangs up the call and starts detecting its current position. After finding it, it sends the data to the caller's number. The caller's number must be displayed correctly - it cannot be hidden, and must be on the list in the GSM locator's memory [13]. 


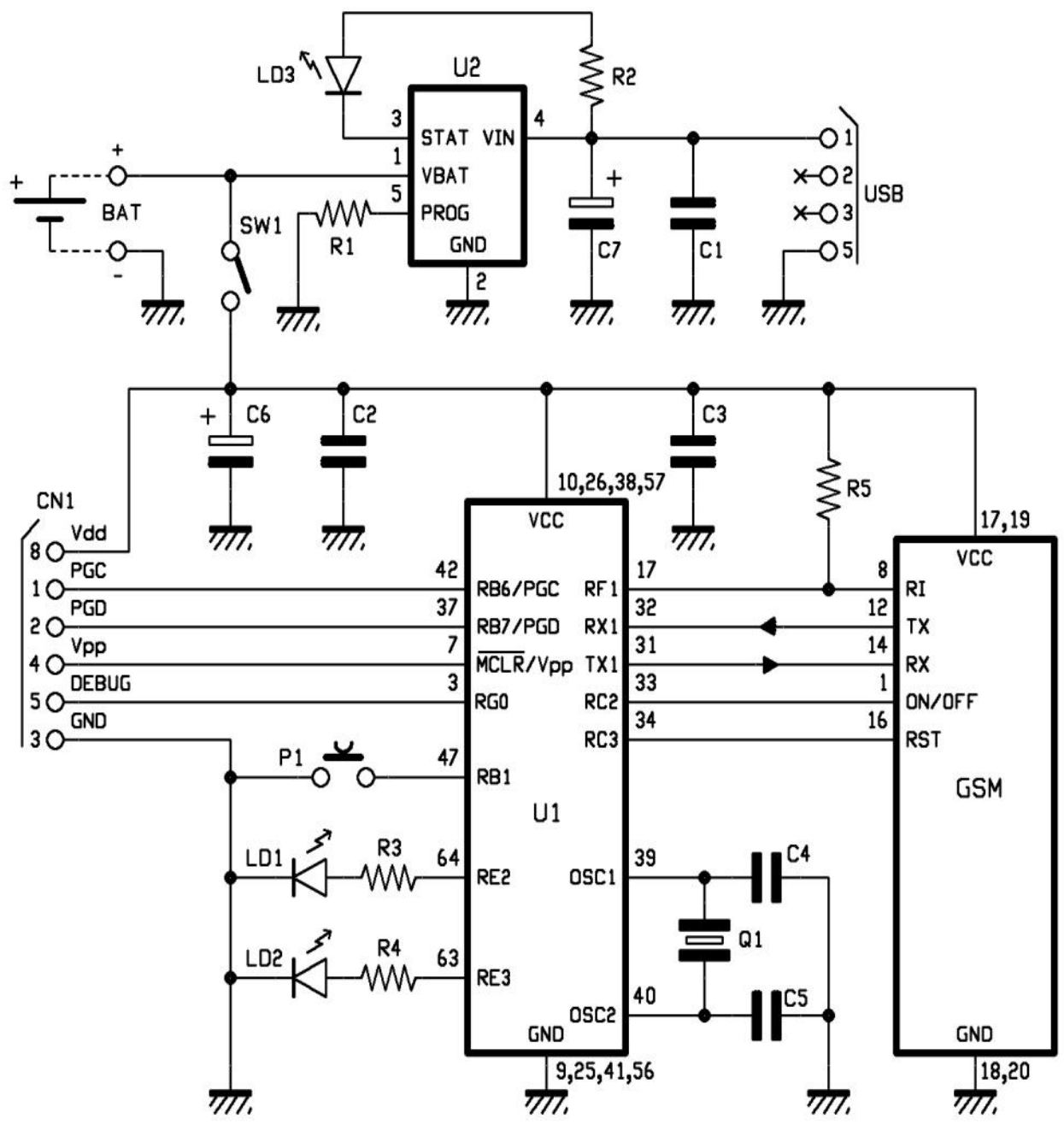

Fig. 7. Control circuit scheme [13]

- Incoming SMS messages. The device, when receiving SMS messages, distinguishes whether it is a configuration SMS message or a request for it to send its current location [14].

- Pressing the SOS button. This feature is optional, and allows the sending of the current location after being pressed. Its practical use is only for tracking people. The SOS button cannot be used to secure a bicycle, only for testing purposes [13].

- The last way of determining the location is automatic initialisation. This can be set with a view to the time parameter, as well as to change the location of the device. The GSM module does not contain a gyroscope, but recognises changes in location according to changes in the actual BTS station [8].

\subsection{GSM locator configuration}

All configuration of the GSM module is done through sending SMS messages to the GSM module. Table 1 provides an overview of possible settings.

\section{Testing the proposed GSM locator}

The GSM locator was tested in two ways. In the first case, the locator was sited on moving object (an automobile) and the reliability of the locator was determined. In the second, the test was performed on a randomly selected location, i.e. the GSM locator had a fixed location.

\subsection{Testing the reliability of a moving GSM locator}

The proposed locator was fitted to a car which was moving around in a densely-settled area and then in an area with low population density. Fig. 8 depicts the testing of the locator in a heavily-settled area. 


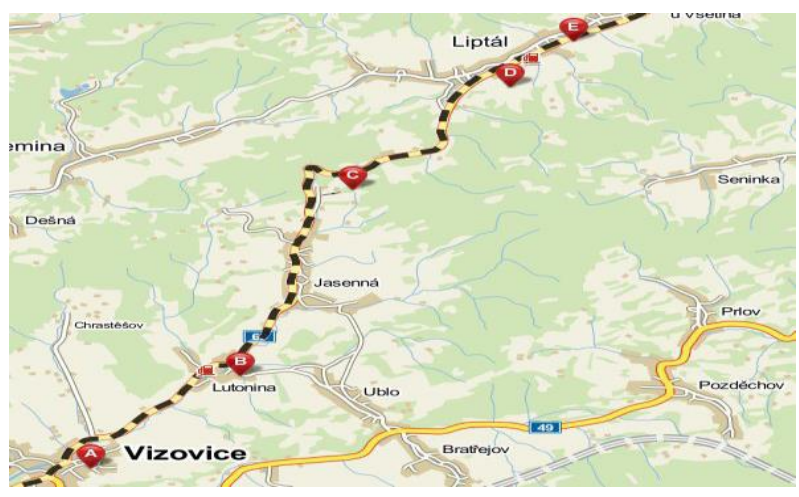

Fig. 8. Map of a high-density settlement area

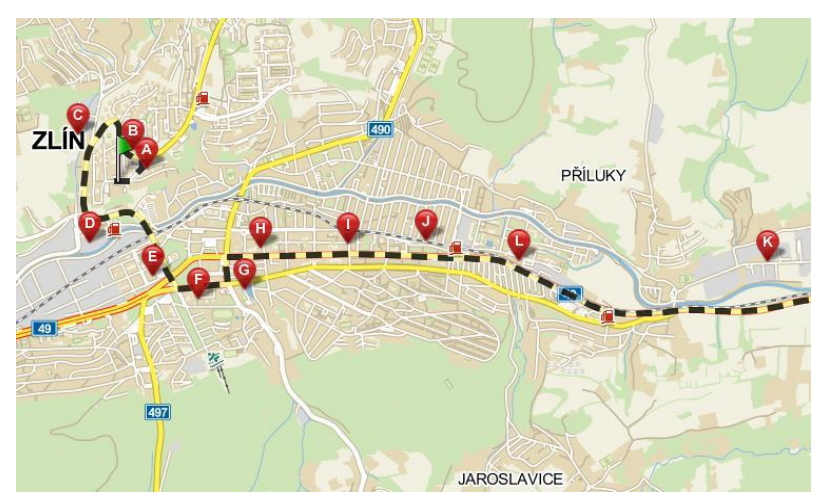

Fig. 9. Map of a thinly-settled area

The dashed line represents the locator's movements, the points designated by the letters A through $\mathrm{K}$ are then, the evaluated positions of the locator. When testing the locator in a heavily-settled urban environment, the locator's imprecision was greater - even reaching $150 \mathrm{~m}$ between the actual position of the locator and its evaluated positions. In areas with fewer buildings, the location evaluations precision was much better; in this case, the difference between the locator position and its evaluation was less than $30 \mathrm{~m}$. A sample locator test is presented in Fig. 9.

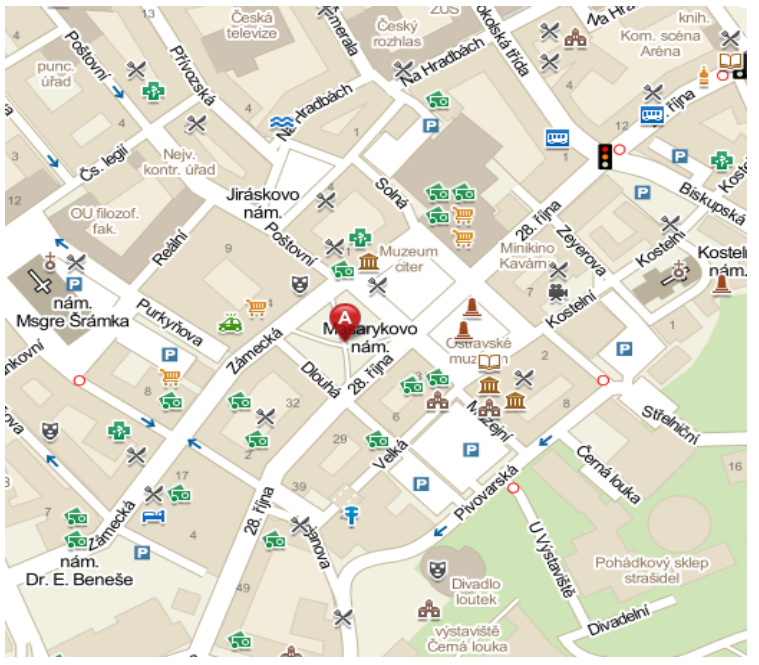

Fig. 10. Random location test

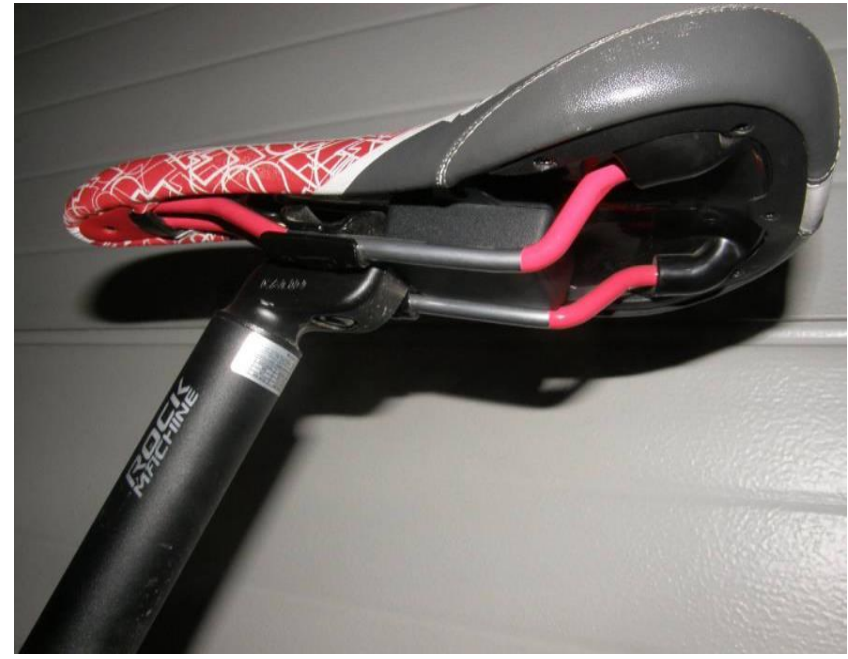

Fig. 11. Installation of the GSM

\subsection{Testing the GSM locator's reliability while determining random locations}

In view of the fact that the GSM locator can be used for securing articles against theft, it was necessary to evaluate the locator's inaccuracy in the case where the locator is a long time in the same location, i.e. does not alter its position. In this case, the search for GSM locator locations was with the aid of SMS messages. In this case, the difference between the actual location of the locator and the evaluated location was no greater than $25 \mathrm{~m}$.

\section{The Evaluation of GSM localisation benefits for security applications}

GSM localisation technology provides the possibility of localisation in areas where a GPS signal is unavailable - especially in dense urban areas without a direct line of sight to satellites. In contrast, in the remote places of our planet, GSM localisation is impossible, or very inaccurate. The possibility of localisation using GSM networks in buildings is the greatest benefit of this technology. Combined with low battery capacity demands and low cost, a GSM locator has the possibility of succeed in the locator market - especially for securing items.

One of the biggest disadvantages of this technology includes its inaccuracy in determining locations. Given that it is impossible to predict where stolen articles fitted with a GSM locator can end up, we cannot rely on an acceptable level of tolerance which the device indicates in densely populated areas. Unfortunately, when using a GSM locator, it can happen that when the device sends us information about its location this is so distorted that localisation is virtually impossible.

The GSM locator presented in this paper was designed with the intent to protect bicycles against theft. The locator can be placed under the seat, where both types of GSM locator can be placed. The first type of locator has a built-in battery for its operations; with the second type, the battery is located in the saddle tube, which holds the seat. 


\section{Acknowledgements}

This work was supported by the Ministry of Education, Youth and Sports of the Czech Republic within the National Sustainability Programme project No. LO1303 (MSMT-7778/2014).

\section{References}

[1] P. Moos, T. Zelinka, V. Malinovsky, Telecommunication services. Prague: CVUT, 2007, ch. 3.

[2] P. Brabenec. Principles of operation of the GSM network. 2013. [Online]. Available: http://www.brabenecz.wz.cz/principy_fungovani_gsm.htm

[3] O. Dudek.. Structure of the GSM network. [Online]. 2015 Available: http://radio.feld.cvut.cz/personal/mikulak/MK/MK05_semestralky/ Struktura_GSM_Ondrej_Dudek.pdf

[4] S. Shafi. SIM card. 2015. [Online]. Available: http://uthmag.com/wp-content/uploads/2012/07/SIM-Cards.jpg

[5] K. Karanoka. WAP push process. 2014. [Online]. Available: http://upload.wikimedia.org/wikipedia/commons/f/f2/WAP_Push_Process.jpg

[6] V.P. Andreeva, K.B. Kirsanovb, P.F. Pleteneva, Yu.V. Poduraeva, V.E. Pryanichnikova, E.A. Prysev: "Technology Supervisory Control for Mechatronic Devices via the Internet", in Journal Procedia Engineering, ISSN: 1877-7058, volume 100, 2015, pp 33 - 40.

[7] A. García-Domínguez, J. Claver, A.M. Camacho, M.A. Sebastián: "Comparative Analysis of Extrusion Processes by Finite Element Analysis”, in Journal Procedia Engineering, ISSN: 1877-7058, volume 100, 2015, pp 74 - 83

[8] M. Orlich. The localization method in GSM. 2014. [Online]. Available: http://access.feld.cvut.cz/view.php?cisloclanku=2006022801

[9] L. Enfora. Enfora enabler IIIG. 2014. [Online]. Available: http://www.farnell.com/datasheets/876254.pdf.

C. Srenk. Localizzatore tramite rete GSM. 2015. [Online]. Available: http://www.futuraelettronica.net/pdf_ita/7100-FT833M.pdf

[10] J. Novak. GSM localization. 2014. [Online]. Available: https://www.t-mobile.cz

[11] K. Rak. PHPBB group. 2014. [Online]. Available: http://www.futurashop.it/forum_/

[12] M. Pospisilik, L. Kouril, I. Motyl, M. Adamek, "Single and double layer spiral planar inductors optimisation with the aid of self-organising migrating algorithm", in Proc. 11th WSEAS International Conference on Signal Processing, Computational Geometry and Artificial Vision. Venice, 2011, pp. 104 - 108.

[13] M. Matysek, M. Adamek, M. Mihok, "Monitoring of Active Devices in Computer Networks. In Annals of DAAAM International 2014. Vienna: DAAAM International Vienna, 2014. ISSN 2304-1382. ISBN 978-3-90150999-5.

[14] M. Pospisilik, L. Kouril, I. Motyl, M. Adamek, "Switching power supply for an autonomous monitoring system”, in Proc. WSEAS International Conference on Systems: Latest Trends on Systems. Greece, 2010. pp. 431-434. 